\title{
Measurement of the Tau properties at LEP
}

\section{Pilar Casado*}

UAB/IFAE, Edifici Cn, E-08193 Bellaterra, Barcelona

E-mail: 'casado@ifae.es!

ABstract: The final results on the tau polarisation measured by the four LEP experiments are presented here. In addition, a preliminary combination of these results taking into account correlated errors for the first time is given. The $\tau$ weak dipole moments, the Michel parameters and the $\tau$ lifetime are also reviewed.

\section{Introduction}

Each of the $\tau$ leptons produced in $e^{+} e^{-} \rightarrow \tau^{+} \tau^{-}$decays in the LEP environment. This important property allows the measurements considered in this paper: weak dipole moments, longitudinal polarisation, Michel parameters and $\tau$ lifetime. The $\tau$ properties are covered in the preceding order, and the conclusions are presented at the end.

\section{Weak dipole moments}

The weak dipole moments of the $\tau$ are the tensorial couplings of the $Z \tau^{+} \tau^{-}$vertex. They are zero at first order in the Standard Model (SM). However, radiative corrections in the SM predict small contributions for these terms [i]1]. Two types of weak dipole moments can be distinguished: the magnetic term, $\mu_{\tau}$, and the $\mathrm{CP}$-violating electric term, $d_{\tau}$. Considering these terms in addition to the SM couplings $v_{\tau}$ and $a_{\tau}$, the $Z \tau^{+} \tau^{-}$current is

$$
\Gamma_{\tau}^{(Z)}=i e\left[v_{\tau} \gamma^{\mu}-a_{\tau} \gamma^{\mu} \gamma_{5}+i \frac{\mu_{\tau}}{2 m_{\tau}} \sigma^{\mu \nu} q_{\nu}+\frac{d_{\tau}}{2 m_{\tau}} \gamma_{5} \sigma^{\mu \nu} q_{\nu}\right]
$$

With this current and the SM currents for the other vertexes, the differential cross-section for $e^{+} e^{-} \rightarrow \tau^{+} \tau^{-}$with unpolarised beams can be expressed as [2]

$$
\frac{d \sigma}{d \cos _{\theta_{\tau}}}\left(\vec{s}_{1}, \vec{s}_{2}\right)=R_{00}+\sum_{i=1,3} R_{i 0} s_{1}^{i}+\sum_{j=1,3} R_{0 j} s_{2}^{j}+\sum_{i, j=1,3} R_{i j} s_{1}^{i} s_{2}^{j} .
$$

\footnotetext{
${ }^{*}$ Speaker.
} 
$R_{i j}$ are functions of the fermion couplings and $\theta_{\tau}$; and $\vec{s}_{1}$ and $\vec{s}_{2}$ are unit vectors for measuring the spin of the $\tau^{+}$and the $\tau^{-}$, respectively, in their rest frames. Some $R_{i j}$ are well known. For example, $R_{00}=$ $d \sigma / d \cos _{\theta_{\tau}}$ (the angular differential crosssection), and $\left(R_{03}\right)_{+} / R_{00}=P_{\tau}$ (the longitudinal $\tau$ polarisation ), taking the $z$ axis parallel to the $\tau^{-}$direction and defining $\left(R_{i j}\right)_{+/-} \equiv\left(R_{i j}+/-R_{j i}\right)$. Assuming the $z$ axis along the $\tau^{+}$direction and the $z-y$ plane containing the $e^{+}$, the four most sensitive terms to the weak dipole moments are $\left(R_{02}\right)_{+},\left(R_{31}\right)_{+},\left(R_{01}\right)_{-}$ and $\left(R_{32}\right)_{-}$. The four second most sensitive terms to the weak dipole moments are $\left(R_{32}\right)_{+},\left(R_{01}\right)_{+},\left(R_{31}\right)_{-}$and $\left(R_{02}\right)_{-}$, taking into consideration that $a_{l}>>v_{l}$.

The ALEPH experiment has updated its measurement of $\operatorname{Re}\left(d_{\tau}\right)$ and has
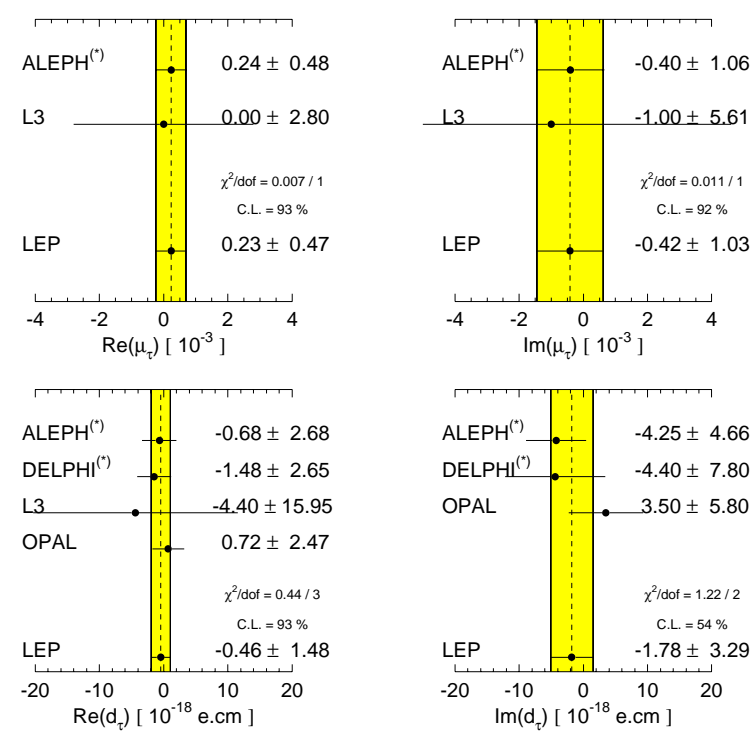

Figure 1: New ALEPH results on the weak dipole moments, previous results of the other LEP experiments, the new LEP averages and the $\chi^{2} /$ dof of the combinations. The symbol $\left.{ }^{*}\right)$ means preliminary result. measured the other weak dipole moments for the first time $[\overline{3 i]}$. The following terms are used for the first time with respect to any preceding analysis of the weak dipole moments: $\left(R_{32}\right)_{+}$(second order term), $\left(R_{31}\right)_{+}$(first order term $)^{1},\left(R_{31}\right)_{-}$(second order term). The ALEPH analysis also considers $\left(R_{02}\right)_{-}$ (second order term) for the first time at LEP. Fig. Iil shows the results of this analysis and the previous published and preliminary results of the other LEP experiments $[-15]$.

\section{Longitudinal polarisation}

\begin{tabular}{|l||cc|cc|cr|cr|}
\hline \multicolumn{1}{|c||}{} & \multicolumn{2}{c|}{ ALEPH } & \multicolumn{2}{c|}{ DELPHI } & \multicolumn{2}{c|}{ L3 } & \multicolumn{2}{c|}{ OPAL } \\
& $\delta A_{\tau}$ & $\delta A_{e}$ & $\delta A_{\tau}$ & $\delta A_{e}$ & $\delta A_{\tau}$ & $\delta A_{e}$ & $\delta A_{\tau}$ & $\delta A_{e}$ \\
\hline \hline ZFITTER & 0.0002 & 0.0002 & 0.0002 & 0.0002 & 0.0002 & 0.0002 & 0.0002 & 0.0002 \\
\hline$\tau$ br. ratios & 0.0003 & 0.0000 & 0.0016 & 0.0000 & 0.0007 & 0.0012 & 0.0011 & 0.0002 \\
\hline Bhabha backg. & 0.0000 & 0.0000 & 0.0012 & 0.0000 & 0.0000 & 0.0000 & 0.0000 & 0.0000 \\
\hline 2-photon backg. & 0.0000 & 0.0000 & 0.0005 & 0.0000 & 0.0007 & 0.0000 & 0.0000 & 0.0000 \\
\hline $\begin{array}{l}\text { radiat. correct. } \\
\& a_{1} \text { modelling }\end{array}$ & 0.0012 & 0.0008 & 0.0010 & 0.0000 & 0.0010 & 0.0001 & 0.0025 & 0.0005 \\
\hline
\end{tabular}

Table 1: Most relevant common systematic errors on $A_{\tau}$ and $A_{e}$ for the four LEP experiments.

The longitudinal polarisation of the $\tau, P_{\tau}$, is a precision electroweak observable measured at LEP. It provides an accurate determination of the effective weak mixing angle

\footnotetext{
${ }^{1}$ The use of $\left(R_{31}\right)_{+}$was suggested in [4].
} 
$\sin ^{2} \theta_{W}^{e f f} . P_{\tau}$ depends on the $\tau$ production polar angle $\theta_{\tau^{-}}$as follows

$$
P_{\tau}=-\frac{A_{\tau}\left(1+\cos ^{2} \theta_{\tau^{-}}\right)+2 A_{e} \cos \theta_{\tau^{-}}}{\left(1+\cos ^{2} \theta_{\tau^{-}}\right)+\frac{8}{3} A_{f b} \cos \theta_{\tau^{-}}}
$$

with $A_{l}=2 v_{l} a_{l} /\left[\left(v_{l}\right)^{2}+\left(a_{l}\right)^{2}\right], v_{l} / a_{l}=$ $1-4 \sin ^{2} \theta_{W}^{e f f}$ and $A_{f b}$ being the forwardbackward charge asymmetry of $\tau$ production.

The four LEP experiments use kinematic distributions of the $\tau$ decay products to measure the polarisation as a function of $\cos \theta_{\tau^{-}}$. Data were collected during the $1990-95 Z$ running period. The decay mode considered are $e \nu \bar{\nu}, \mu \nu \bar{\nu}$,

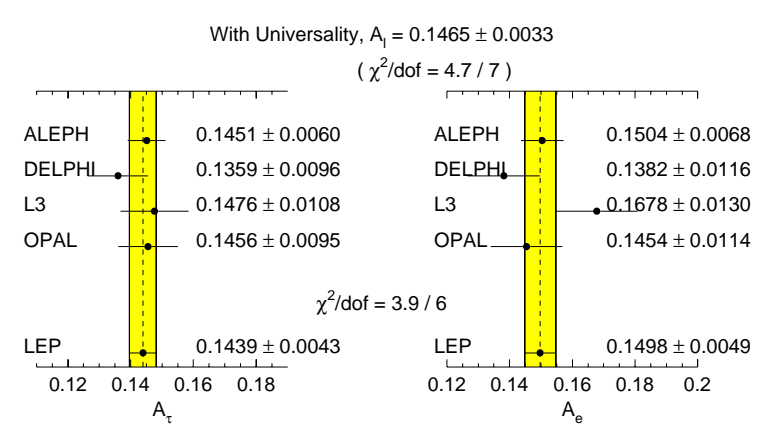

Figure 2: New final results of ALEPH and OPAL on $A_{\tau}$ and $A_{e}$, and the published results of DELPHI and L3. The new LEP results on $A_{\tau}$ and $A_{e}$ and the $\chi^{2} /$ dof are also shown. On the top of the figure, the LEP fit for $A_{l}$ assuming lepton universality is also presented. $\pi \nu, \rho \nu, a_{1}(3 \pi) \nu$ and $a_{1}\left(2 \pi^{0} \pi\right) \nu$.

The method of measuring $P_{\tau}\left(\cos \theta_{\tau^{-}}\right)$yields nearly independent determinations of $A_{\tau}$ and $A_{e}$. Thus, the $\tau$ polarisation measurements provide both a determination of $\sin ^{2} \theta_{W}^{e f f}$ and a test of the hypothesis of universality of the $Z$ couplings to the electron and the $\tau$.

ALEPH and OPAL have presented new final results on the $\tau$ longitudinal polarisa-

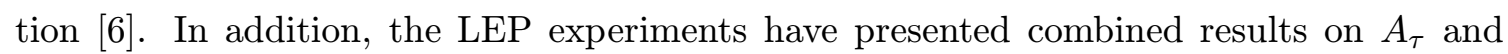
$A_{e}$ taking into account correlated systematic uncertainties for the first time [i] relevant common systematic errors are shown in Table

Fig. $\overline{2}$ shows the new final results of ALEPH and OPAL on $A_{\tau}$ and $A_{e}$, and the

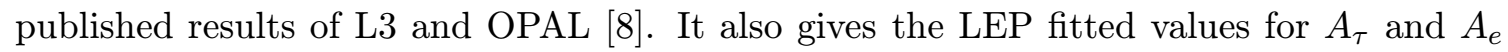
with no assumption on lepton universality, and $A_{l}$ assuming $e-\tau$ universality. This last result provides a value of

$$
\sin ^{2} \theta_{W}^{e f f}=0.23159 \pm 0.00041
$$

\section{Michel parameters}

The $\tau$ Michel parameters allow to test possible deviations from the SM description in the $\tau$ decay. In $\tau \rightarrow l \nu_{\tau} \overline{\nu_{l}}$, the coupling constants can be experimentally accessed via the energy spectra of the daughter leptons. If the polarisation of the daughter leptons is not known, the spectrum of the $\tau$ decays can be parametrised at Born level by

$$
\frac{1}{\Gamma} \frac{d \Gamma}{d x_{l}}=h_{0}\left(x_{l}\right)+\eta h_{\eta}\left(x_{l}\right)+\rho h_{\rho}\left(x_{l}\right)-P_{\tau}\left[\xi h_{\xi}\left(x_{l}\right)+\xi \delta h_{\xi \delta}\left(x_{l}\right)\right],
$$

where the coefficients $\eta, \rho, \xi$ and $\xi \delta$ are the Michel parameters, the dependence of the $h$ functions on $x_{l}$ is known, $P_{\tau}$ is the average $\tau$ polarisation and $x_{l}=E_{l} / E_{\text {beam }}$. In The SM, $\eta=0, \rho=0.75, \xi=1$ and $\xi \delta=0.75$. 
Fig. 'Bי' shows the recently published results of ALEPH on the Michel parameters $[\overline{9} \overline{9}]$ and the results of the other LEP experiments [i] $\left.{ }_{1}^{1} \overline{1}\right]$. The LEP average is also given.

\section{Lifetime}

The $\tau$ lifetime can be used to test lepton universality together with the $\tau$ leptonic branching ratios, $B_{e}$ and $B_{\mu}$, and the measurement of $m_{\tau}$ by

$$
\begin{aligned}
& \left(\frac{g_{\tau}}{g_{\mu}}\right)^{2}=0.9996 \frac{\tau_{\mu}}{\tau_{\tau}} \frac{m_{\mu}^{5}}{m_{\tau}^{5}} B_{e}, \\
& \left(\frac{g_{\tau}}{g_{e}}\right)^{2}=0.9996 \frac{\tau_{\mu}}{\tau_{\tau}} \frac{m_{\mu}^{5}}{m_{\tau}^{5}} \frac{B_{\mu}}{f\left(m_{\mu}^{2} / m_{\tau}^{2}\right)},
\end{aligned}
$$

where the numerical factor accounts for electroweak propagator and radiative corrections in the $\tau$ and $\mu$ decays. The function $f$ is a known phase space factor with
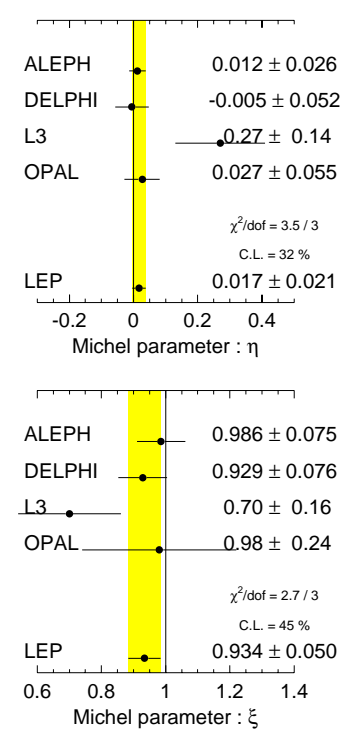
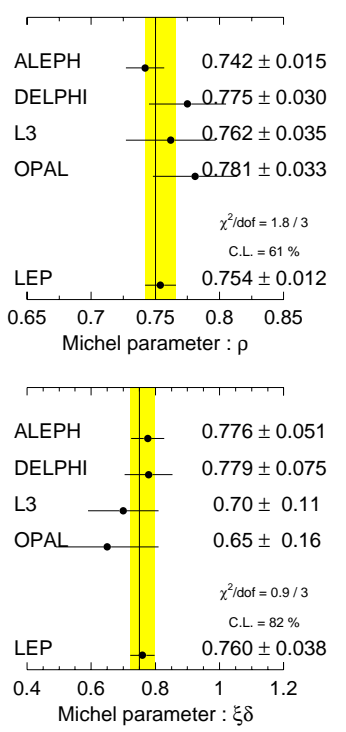

Figure 3: New final measurements of ALEPH on the Michel parameters, the results of the other LEP experiments, the new LEP average and the $\chi^{2} / d o f$ of the combination. The SM expectation is shown as a vertical line for each parameter. value $f\left(m_{\mu}^{2} / m_{\tau}^{2}\right)=0.97256$.

The DELPHI collaboration has presented a new preliminary result of the $\tau$ lifetime [i] 1 in which is shown on the top part of Fig. 迹 together with the previous results of the other LEP experiments and the new LEP average. On the bottom part of this figure, the new LEP $\tau$ lifetime is plotted versus the LEP values of $B_{e}$ and $B_{\mu}\left[{ }_{1}^{1} \overline{2} \overline{2}\right]$. The diagonal band of these figures represents the SM prediction according to the previous equations, with the width reflecting the experimental uncertainty in $m_{\tau}$. Taking the PDG values for $m_{\mu}, m_{\tau}$

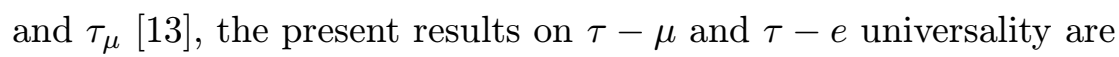

$$
\begin{aligned}
& \left(\frac{g_{\tau}}{g_{\mu}}\right)=0.9999 \pm 0.0023, \\
& \left(\frac{g_{\tau}}{g_{e}}\right)=1.0002 \pm 0.0023,
\end{aligned}
$$

both consistent with the SM expectation.

\section{Conclusions}

ALEPH and OPAL have presented new final results on the measurement of the $\tau$ polarisation. The four LEP experiments have combined their results on the $\tau$ polarisation taking into account common errors for the first time. This yields a precise measurement of the effective weak angle of $\sin ^{2} \theta_{W}^{e f f}=0.23159 \pm 0.00041$, in good agreement with other leptonic contributions to $\sin ^{2} \theta_{W}^{e f f}[\underline{i}]$. 
The Lorentz structure of both the neutral current and the $\tau$ decay have been revised, with no deviation from the SM expectations in both cases. ALEPH has presented new preliminary results on the tau weak dipole moments and new final results on the Michel parameters.

Finally, a new preliminary measurement of the $\tau$ lifetime has been presented by DELPHI, allowing to set new LEP values on $\tau-\mu$ and $\tau-e$ universality. The new universality ratios agree with the SM prediction at the few per mil level.

\section{Acknowledgements}

I wish to thank Enrique Fernández, Henri Videau, Roberto Tenchini and Manel Martínez, for their useful comments and help on the accomplishment of this paper.
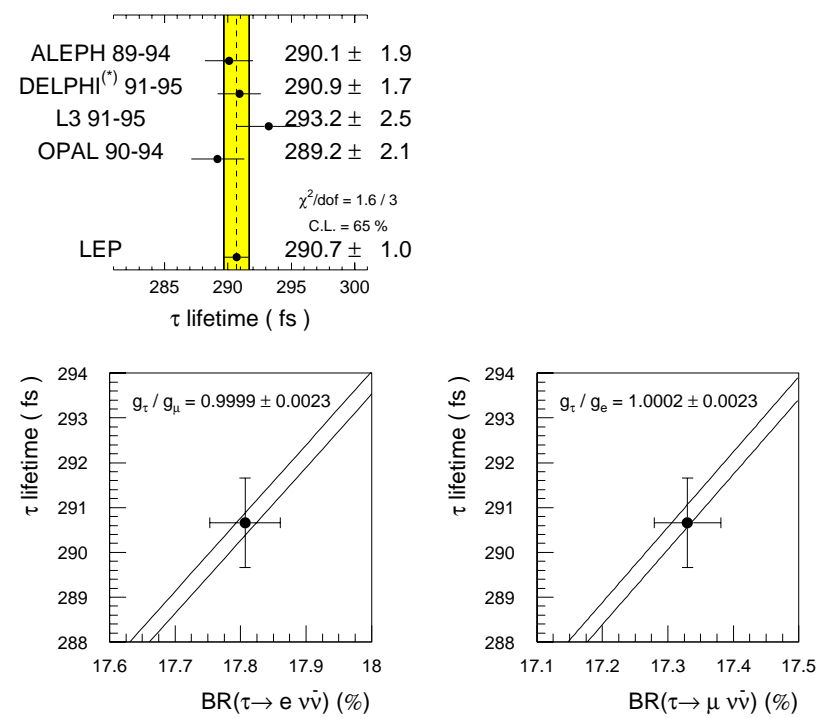

Figure 4: On the top plot, new DELPHI result on the $\tau$ lifetime, the results of the other LEP experiments, the new LEP average and the $\chi^{2} /$ dof of the combination. The symbol ${ }^{(*)}$ means preliminary result. On the bottom plots, the new LEP $\tau$ lifetime versus the LEP values of $B_{e}$ and $B_{\mu}$.

\section{References}

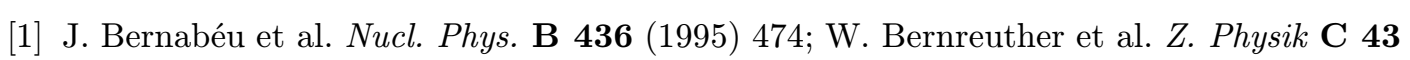
- -

[2] U. Stiegler 'ZZ Physik

[3] ALEPH Coll. Contrib. Paper for EPS HEP 2001 (Budapest) and LP01 (Rome) (Abstr. 224)

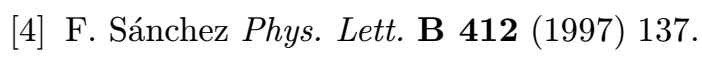

[5] A. Zalite Nucl. Phys. (Proc. Suppl.) B 76 (1999) 229.

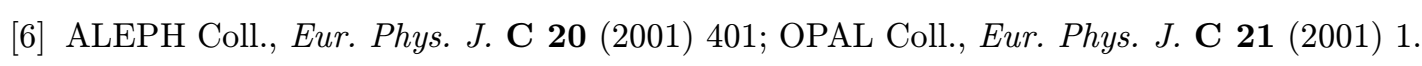

[7] Private comun. with the LEP Electroweak Working Group.

[8] DELPHI Coll., 'Z. Physik

[9] ALEPH Coll. CERN EP/2001-035, submit. to Eur. Phys. J. C.

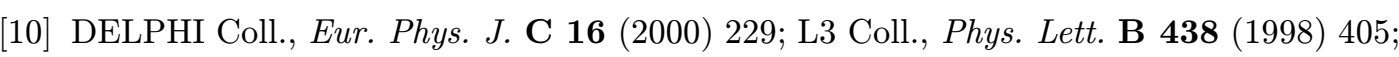
OPAL Coll. 'Eur. Phys.

[11] DELPHI Coll. Contrib. Paper for EPS HEP 2001 (Budapest) and LP01 (Rome) (Abstr. 339).

[12] S. H. Robertson Nucl. Phys. (Proc. Suppl.) B 98 (2001) 67.

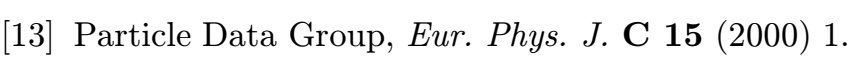

